\title{
ADDRESS BY THE RETIRING PRESIDENT :* SOME PROBLEMS IN WIRELESS TELEGRAPHY
}

\author{
By Prof. H. M. Macdonald.
}

[Read November 14th, 1918.]

THE aim of mathematical physics is the application of mathematics to the phenomena of physics to obtain an intelligible representation of these phenomena. Different types of problems can be recognised and of these the first is the selection of the particular problem which promises to admit of successful mathematical treatment, and at the same time to preserve the most essential features of the phenomena to be represented. This involves the careful comparison of the available observations connected with the phenomena under consideration, with the view of ascertaining the most outstanding resemblances between these observations, and thence deducing the most likely underlying physical source of the phenomena. The problem having been strictly defined on the physical side, the next step is to choose the geometrical setting; and if the results obtained by the solution of the problem thus selected are in sufficient agreement with the observations, the primary problem can be regarded as solved. There then remain the other types of problems to be solved, viz. those obtained by varying the geometrical setting and those that result from taking into account other physical causes. In illustrating the foregoing remarks the problems of electrostatics may be cited. The primary problem is that of the perfectly conlucting sphere in an indefinitely extended vacuum. When the solution of this problem had been obtained, the next object of investigation was the effect on the result of altering the shape of the conducting body, and this led to the discussion of the case of the ellipsoid, the circular disc, a body with a sharp edge such as a spberical bowl, a ring shaped body such as an anchor ring, and so on. There are further the problems which result from substituting for the perfectly conducting body an imperfectly conducting body or re-

* The publication of this address, delivered at the Amnual General Meeting of November 14th, 1919, has been unavoidably delayed. 
placing the vacuum outside the body by some dielectric medium either homogeneous or non-homogeneous.

The form in which the solution of a mathematical physical problem is presented is of considerable importance, when it is remembered that comparison has to be made with the results of observation; and the ideal solution is one which gives a simple picture of the phenomena and at the same time admits of rapid reduction to numerical units. The problem of the point charge outside a perfectly conducting sphere affords a perfect example, the effect of the induced distribution in the sphere being equivalent to that of a point charge placed at a definite point inside the sphere.

The particular problems which it is proposed to examine from the above point of view are those of wireless telegraphy, viz. the problems of the emission of electric radiation from a sending station, its reception at another station, and of its transmission from the one to the other.

There are three distinct types of problems connected with the oscillation of a vibrating system; the case of a vibrating system from which there is no loss of energy; the case of a vibrating system from which energy is being radiated freely; and the case of a vibrating system through which the loss of energy by radiation is being made good at exactly the same rate as the energy is being radiated from the system, so that the radiation is steady. Examples of the three different types are the vibration of a gas in the space bounded by a closed surface such as a sphere; the radiation from a nearly closed surface, such as a sphere with an aperture in the surface, of the energy of a disturbance set up in the air inside; and the radiation from such a space when the energy inside is being maintained. The first problem has been completely solved in the case of the sphere; and the result is that any possible system of vibration in the space can be expressed in terms of certain normal types unlimited in number, any one of which can exist separately. The problem of free radiation from the space such as that inside a spherical bowl has not been solved, but it can be predicted that the solution will involve oscillations of different periods and different rates of lecay, and further that the oscillations of a particular period cannot necessarily exist separately.

If in the case of the spherical bowl the aperture is small, the periods of the different oscillations will differ but slightly from the periods of oscillations of the air in the closed sphere, and the rates of decay will be small; but it may be expected that, as the aperture is increased in size, the periods will differ more and more from those of the oscillations in the closed space, and at the same time the rates of decay will increase.

The problem of a radiating system, when the loss of energy is being 
replaced so that the radiation is steady, admits of being solved at least approximately in several cases, for example the case of the sphere with a circular aperture. The problem to be solved in such a case is that of finding the periods of the oscillation which are most easily maintained, i.e. the periods of resonance; and if the aperture is small the periods will differ but slightly from those of the free oscillations in the closed space, but as the aperture is increased the periods will differ appreciably both from those of the free oscillations in the closed spaces and those of the second problem.when the energy is being freely radiated without being replaced. The difference between the periods in the three cases can be illustrated from the case of a simple vibrating system with one period $2 \pi / n$ when there is no decay of energy; if this system loses energy and the rate of decay is $m$, the period of the free oscillation is $2 \pi / \sqrt{ }\left(n^{2}-1+m^{2}\right)$, and the period of the oscillation to which the system resonates is $2 \pi / \sqrt{ }\left(n^{2}-\frac{1}{2} m^{2}\right)$, from which it appears that when the rate of decay is appreciable the difference between the corresponding periods may be considerable. It should be observed that in the case of maintained oscillations the oscillations of a particular period can be treated separately. It should not however be concluded that any radiating system can act effectively as a resonator ; certain conditions have to be satisfied. Taking the case of the sphere with an aperture, it is clear that, if the energy of the oscillation is maintained by a source placed inside the sphere, it would be po:sible to replace the single aperture by a number of apertures in the surface, the total radiation outwards remaining the same; but, if the source maintaining the oscillations is outside the sphere, the effects of the different apertures will not generally reinforce each other, but will interfere, and resonance will not take place. A necessary condition therefore for effective resonance is that the radiating system which is to be used as a resonator is such that the radiation given out by it is concentrated. Further a radiating system which is to be effective as a source for the emission of radiation must be one for which the rate of decay is appreciable.

These two conditions must be satisfied by any electrical system which is to be efficient for the emission and detection of electrical radiation, and it is therefore necessary to consider the problem of rediation from a conducting body with these conditions in view. The problem of radiation from a perfectly conducting sphere has been completely solved, and taking the simplest case, viz. that in which the initial electrical distribution on the surface is specified by a spherical harmonic of the first order, it appears that in the immediate neighbourhood of the surface the transfer of energy outwards from the sphere takes place from the equator. This result ad- 
mits of generalisation for the case of any perfectly conducting body; for the electrical force is everywhere normal to the surface of the body at the surface, and therefore the energy in the immediate neighbourhood of the surface can only flow along the surface at any place where the electric force is finite; hence the energy in the immediate neighbourhood of the surface can only leave the. surface at places where the electric force yanrshes. Applying this to the case of an ovary ellipsoid of revolution, the initial distribution being one which vanishes only at the equator, the radiation will always take place from the equator; and as the ellipsoid approaches to the form of a straight rod, terminated at both ends, the wave length of the oscillations approximates to double the length of the rod, while at the same time the rate of decay of the oscillations tends to zero. It therefore follows that a straight conducting rod for which the conditions presupposed in this solution, viz. that the surrounding medium can support the electric forces everywhere at the surface, are satisfied, cannot be effective for the emission or detection of oscillations. It should however be observed that as the ellipsoid approaches the form of a straight rod the amplitude of the electric force in the immediate neighbourhood of the ends increases, ultimately being indefinitely great; and when the surrounding medium is air it may be expected that, as in the corresponding case of a charged conductor with a sharp point or edge, electric discharge will take place from the ends. This was first observed by Sarasin and Birkeland, and an examination of their observations shows that radiation is taking place from the end of the wire. An exact solution of the problem of radiation from a freely radiating perfectly conducting straight rod would require a knowledge of the mechanism of the discharge at the onds which is not so far available; but if, as in the experiments above referred to, the energy radiated away is replaced so that the radiation is steady, the flow of energy outwards from the rod cannot differ essentially from the flow from a simple electric oscillator. The problem then admits of solution, and the result is that the wave length of the oscillation of longest period is two and a half times the length of the rod. The determination of the wave length of the radiation from a straight rod has been the subject of experimental investigation by a number of different observers, who have obtained results which range from double the length of the rod to two and a half times its length; but an examination of the conditions, where these have been sufficiently detriled, would seem to show that these differences are to be accounted for by the fact that the arrangements are different snd that different phenomena are being observed.

In some of the experiments the arrangements are clearly such that the exergy associated with the distribution which has been set up on the rod 
by the external oscillation is practically being freely radiated away, and therefore the observed wave length is neither that which corresponds to steady radiation, when the energy is maintained, nor that which corresponds to the case of a rod where the surrounding medium is such that no radiation takes place from the ends. In other cases the arrangements wre such that radiation from the ends is prerented, as for example when the rod is immersed in some non-conducting oil.

The wave length corresponding to the other possible periods are readily obtained; and it is important to observe that in these cases the distance between successive nodes along the rod is the wave length of the oscillations in question, a result which has been verified for wires by various observers. The solution of the problem for the case of an imperfectly conducting rod can be obtained; and the result is that (if the specific resistance can be assumed to be approximately the same in the case of oscillation as that for metallic conductors in which there are steady currents) the relation between the wave lengths of the oscillation and the length of the rod only differs by very small quantities from the relation between the wave lengths and the length of the rod when the rod is perfectly conducting. This assumes that the magnetic permeability of the material of the rod is the same as that of the surrounding medium or does not differ greatly from it. If the magnetic permeability of the material of the rod is of the same order as that of iron, the difference in the relation would be appreciable. These results are in agreement with observation; in particular it has been observed that for copper wires the difference between successire nodes along the wire is equal to the wave length of the oscillation, while the distance between successive nodes along an iron wire differs from the wave length by an amount which, though small, is nppreciable.

It follows from the above that the difference between the observed wave lengths for the straight rod and double its length cannot be referred to imperfect conductivity for two reasons, viz. that the effect of imperfect conductivity is too small if it is of the same order as in the case of a steady current, and that the distance between successive nodes along the rod does not differ appreciably from the wave length of the oscillations. An idea of the magnitude of the rate of radiation from the rod, when it is radiating steadily, can be obtained by comparing it with a simple vibrating system; in such a system the amplitude of the oscillations would diminish by approximately one-fourteenth for each oscillation when radiating freely, if the relation between the wave length when there is no radiation and when the radiation is steady were as 4 to 5 . Hence it may be concluded that the radiation from the rod is not small; and as, further, this radia- 
tion takes place from the ends, the rod can act effectively both for the emission of radiation and for resonating to radiation from other sources. The simplest arrangement of a sending or receiving station consists essentially of a vertical antenna in which the radiation is emitted or collected by the free end; and the presence of points or angles from which radiation can take place is an essential feature of the arrangements of all wireless telegraph stations. It has been observed that the effective distance of a station depends on the height of the antenna, both at the sending station and at the receiving station; and in particular for the case of umbrella stations that the height which is effective is the height of the extremities of the ribs of the umbrella above the conducting surface, thus showing that the radiation is emitted and received at the extremities of the ribs.

An important problem in this connection is the determination of the wove length of the oscillations which are most effective for transmitting signals. When it is remembered that each signal occupies a time which is very great compared with the period of an oscillation, it is clear that the production of a signal requires a train of waves containing very many oscillations, and, that being so, the problem to be solved is approximately the problem of radiation from the sending station when the radiation is steady; and, as has been seen, this problem admits of solution in certain cases. For example, in the case of the simple vertical antenna, the required wave length is that belonging to a straight conducting rod radiating steadily, the length of the rod being double the height of the antenna, so that the fundamental wave length in this case is five times the height of the antenna. This result agrees with observation. The solution of the problem in more complicated arrangements has not so far been solved, but the same method with the necessary slight modifications would apply.

Difficulties have arisen in connection with the measurements of the effect at a distance from the sending station. It has usually been assumed by observers that the observed disturbance is expressible in terms of the square of the amplitude of the oscillation. The reason for this assumption is not clear; but it may have been suggested by the expression for the intensity of sound or the expectation that it depended simply on the energy. The essential feature of any detecting arrangement would appear to be that the resistance in a portion of a circuit is not constant, but that the resistance diminishes when the electric force increases above a certain magnitude. Accurate information is not available to enable the problem of any particular arrangement being stated in accurate terms, so as to be submitted to analysis; but it is comparatively easy to state a mathematical 
problem which involves the essential fact that the resistance is not constant and which admits of solution. For example, assuming that the resistance is constant when the electric force is less than $E_{1}$, while, when it is greater than $E_{1}$, the change in the conductivity is proportional to its excess over $E_{1}$ when the electric force is in one direction, with corresponding conditions when the electric force is in the other direction, the electric force for which the conductivity begins to vary in this case being $E_{2}$, a relation can be obtained between the resultant current in the detecting circuit and $E$ the amplitude of the electric force in the incident waves. When $E$ is less than $E_{1}$ and $E_{2}$ the resultant current is proportional to $E$, and when $E$ is greater than $E_{1}$ and $E_{2}$, the expression for the resultant current tends to the form $a E+b E^{2}$, ultimately tending to $b E^{2}$.

It follows that the relation between the amplitude of the oscillations and the current in the receiving telephone is in general not a simple relation, although it may be expected that, as in the above formula when $E$ is small, as it will be at a considerable distance, the current in the telephone circuit is approximately proportionally to the amplitude of the electric force. This agrees with the result of observation.

The remaining problems are those connected with the transmission of signals to a distance around the earth's surface. The primary problem in this connection is to select the simplest arrangement which possesses the essential features; and when the portion of the earth's surface that intervenes between the two stations is covered by the sea, the problem is at once simplified by assuming the surface to be perfectly conducting. Further when it is remembered that the electric force is everywhere perpendicular to a perfectly conducting surface, it is clear that the essential features of the problem are preserved if the source of the waves is taken to be a simple oscillator whose axis is perpendicular to the surface. This problem admits of solution, and it has been shown that the results obtained agree with the observed results at a considerable distance. The explanation provided by this theory, known as the diffraction-theory, accounts for the most important features of the transmission of signals. Probably the reluctance to adopt it owes in some measure its origin to comparison with optical phenomena; but it should be observed that as the ratio of the wave length used in wireless telegraphy to the earth's radius is of the order $10^{-3}$, the size of the corresponding sphere in the case of light is indefinitely small, and the observed results in the case of optical phenomena do not provide a true analogy. The remaining problems connected with the transmission of signals are first the effect of imperfect conduction and second the effect of the atmosphere.

In the case of the transmission over the surface of the sea, the effect 
of imperfect conduction is, as might have been expected, negligible, and not greater than the possible errors of observations at the distances involved. With regard to the effect of the atmosphere, there is not at present sufficient detailed observation to enable the problem to be submitted to accurate mathematical analysis. The main question to be answered would appear to be the effect of change of atmospheric conditions at sending stations or at the receiving stations, that is whether the intensity of the radiation from a station depends on the atmospheric conditions at that station. Further there is the question as to whether there is reflection of the waves from the upper atmosphere, and whether there may not be absorption under certain conditions. There are indications that under certain circumstances reflection does take place in the atmosphere, but until more information is available as to the conditions obtaining in the atmosphere at different heights above the earth's surface, an approximate estimate of the effect to be expected cannot be obtained. 